Pacific Journal of Mathematics

POINCARE COBORDISM EXACT SEQUENCES AND CHEER IE 


\title{
POINCARÉ COBORDISM EXACT SEQUENCES AND CHARACTERISATION
}

\section{HimadRi Kumar MukerJee}

\begin{abstract}
Exact sequences connecting oriented and unoriented cobordism groups of Poincaré duality spaces, analogous to Rohlin's and Wall's exact sequences in differential and piecewise linear categories, are established and characterisation of elements of Poincaré cobordism groups (both oriented and unoriented) in terms of spherical characteristic numbers and index are given.
\end{abstract}

Introduction (Notations and Main results). By a Poincaré duality space we will mean a finite complex satisfying Poincaré duality with local coefficients in the sense of [27]. Corresponding to each P.D. space $X^{n}$ of dimension $n$, there is a $k$-spherical fibration $\nu_{X}^{k}(k \gg n$, i.e., $\nu_{X}$ is a stable fibration) unique up to fibre homotopy equivalence, and a commutative diagram:

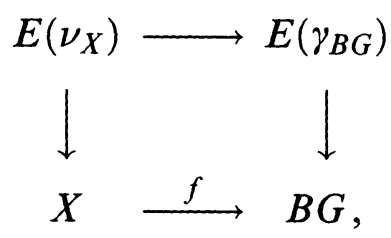

where $\gamma_{B G}: E\left(\gamma_{B G}\right) \rightarrow B G$ is the stable universal unoriented spherical fibration and $f$ is the classifying map of $\nu_{X}$. Let $\gamma_{B S G}: E\left(\gamma_{B S G}\right) \rightarrow$ $B S G$ be the stable universal oriented spherical fibration ( $B S G$ is obtained from $B G$ by killing the first Stiefel-Whitney class). If $\nu_{X}$ can be classified by $\gamma_{B S G}$ then we call $X$ an oriented P.D. space. We now define various Poincaré cobordism groups. Suppose $\eta^{k}: E(\eta) \rightarrow B$ is a $k$-spherical fibration. We define $T\left(S^{n+k}, T\left(\eta^{k}\right)\right)$ to be the set of cobordism classes of triples $\left(X^{n}, f, b\right)$, where $X^{n}$ is a P.D. space and $f, b$ are base and total space maps, respectively, in the following diagram

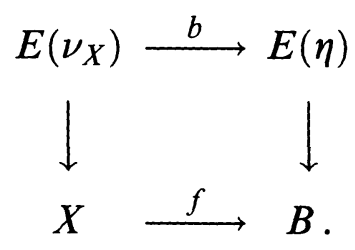


$T(\eta)$ denotes the Thom space of fibration $\eta$ (see $[2,10]$ ). We define $\Omega_{n}^{\text {P.D. }}$ to be $T\left(S^{n+k}, T\left(\gamma_{B S G}\right)\right)=T\left(S^{n+k}, M S G\right)$ and $N_{n}^{\text {P.D. }}$ to be $T\left(S^{n+k}, T\left(\gamma_{B G}\right)\right)=T\left(S^{n+k}, M G\right)$. (We are using here the standard notations $M S G, M G$ for the Thom spaces of $\gamma_{B S G}, \gamma_{B G}$ respectively.) For brevity we shall denote elements of $\Omega^{\text {P.D. }}$ or $N_{*}^{\text {P.D. }}$ by $[X]$ keeping $f, b$ implicit, unless otherwise needed.

Define $\tilde{N}_{n}^{\text {P.D. }}$ to be the cobordism classes of pairs $\left(M^{n}, \alpha\right)$, where $M^{n}$ is a P.D. space and $\alpha$ is a lift of the first Stiefel-Whitney class, $w_{1}(M)$, of $M$ to an integral class; that is, the following diagram is commutative (up to homotopy):

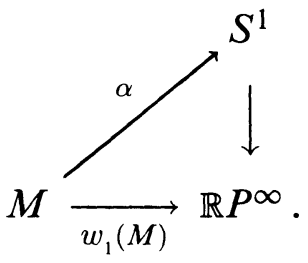

There are natural maps,

(i) $\Omega_{n}^{\text {P.D. }} \rightarrow N_{n}^{\text {P.D. }}$, which is induced by the natural projection $\pi: B S G \rightarrow B G$

(ii) $\tilde{N}_{n}^{\text {P.D. }} \stackrel{\partial}{\longrightarrow} \Omega_{n-1}^{\text {P.D. }}$, which will be constructed in $\S 5$, see $(5.4)$, (5.5), (5.6).

We are now in a position to state our main theorems:

Theorem (A). The sequence

$$
\Omega_{n}^{\text {P.D. }} \stackrel{2}{\longrightarrow} \Omega_{n}^{\text {P.D. }} \stackrel{r}{\rightarrow} N_{n}^{\text {P.D. }}
$$

is exact for all $n>0$, where 2 denotes the homomorphism obtained by taking the disjoint union of two copies of an element of $\Omega_{n}^{\text {P.D. }}$.

THEOREM (B). The long sequence

$$
\rightarrow \Omega_{n}^{\text {P.D. }} \stackrel{2}{\longrightarrow} \Omega_{n}^{\text {P.D. }} \stackrel{r}{\longrightarrow} \tilde{N}_{n}^{\text {P.D. }} \stackrel{\partial}{\longrightarrow} \Omega_{n-1}^{\text {P.D. }} \stackrel{2}{\longrightarrow} \cdots
$$

is exact for all $n \geq 1$.

Before stating the next results let us introduce some terms. Elements of $H^{*}(B G ; \Lambda)$ are called universal spherical $\Lambda$-characteristic classes. The normal spherical $\Lambda$-characteristic classes of a P.D. space $X$ will be the $\Lambda$-characteristic classes of $\nu_{X}$ as induced by $\nu_{X} \rightarrow \gamma_{B G}$. Characteristic numbers of $X$ are defined as usual. 
Theorem (C). An element $[X] \in N_{n}^{\text {P.D. }}$ is zero if and only if the $\mathbb{Z} / 2$-normal spherical characteristic numbers of $X$ vanish.

THEOREM (D). An element $[X] \in \Omega_{n}^{\text {P.D. }}$ is zero if and only if

(i) $n \not \equiv 0(\bmod 4)$ and all $\mathbb{Z} / 2$ as well as integral normal spherical characteristic numbers of $X$ are zero.

(ii) $n \equiv 0(\bmod 4)$ and all $\mathbb{Z} / 2$ as well as integral normal spherical characteristic numbers and index of $X$ are zero.

In the category of Poincare duality spaces, P.D., Theorem (A) represents an analogue of Rohlin's Theorem [18] and Theorem (B) represents an analogue of Wall's theorem [25] giving exact sequences of cobordism groups of differentiable and piecewise linear manifolds. Theorems (C) and (D) characterize Poincare cobordism classes by means of characteristic numbers and index analogous to the theorem of Thom-Milnor-Wall in the category DIFF of differential manifolds (see e.g. [23, 24]).

The main obstacle in the transition from DIFF to P.D. is the absence of transversality principle in the latter category. Levitt's exact sequence [12] measures with sufficient precision this failure of transversality principle in P.D. . So our main task is to examine Levitt's sequence closely while considering Poincaré cobordism groups of dimension $n \geq 3$. For $n=1,2$ direct arguments using classification of Poincaré duality spaces of dimensions 1,2 of $[26,9]$ give us the required results.

The paper is arranged as follows: In $\S 1$ we prove Theorem (A) for $n=1,2$. In $\S 2$ we recall Levitt's exact sequence and prove a fundamental lemma $(2.3)$. In $\S 3$ we prove Theorem $(A)$ for $n \not \equiv 3(\bmod 4)$, $n>3$. In $\S 4$ we prove Theorem $(A)$ for $n \equiv 3(\bmod 4)$. Section 5 is devoted to the proof of the existence of the map $\partial: \widetilde{N}_{n}^{\text {P.D. }} \rightarrow \Omega_{n-1}^{\text {P.D. }}$. In $\S 6$ we prove Theorem (B). Theorems (C) and (D) are proved in $\S 7$. The results of this paper were announced in $[14,15]$.

I am indebted to the referee for his invaluable comments and suggestions, especially in selecting a suitable definition of $\widetilde{N}^{\text {P.D. }}$.

1. Proof of Theorem (A) for $n=1,2$. We first recall the following classification theorems for low dimensional P.D. spaces:

(I.1) Theorem (Wall) [26]. An one-dimensional P.D. space is a homotopy circle. 
(I.2) TheOREM (Eckmann \& others) [9]. A two-dimensional P.D. space is homotopy equivalent to a closed surface of genus $\geq 0$.

With reference to the above theorems we can proceed to prove Theorem (A) for $n=1,2$. The same kind of proof can be given for both $n=1$ and 2 . We instead prefer to give different kinds of proofs for $n=1$ and for $n=2$.

$n=1$. Let $\left[\left(X^{1}, f, b\right)\right]$ be an element of $\Omega_{1}^{\text {P.D. }}$. If $h: S^{1} \rightarrow X^{1}$ is a Wall homotopy equivalence, then the pair $\left(D^{2} \cup_{h} X^{1}, X^{1}\right)$ is homotopy equivalent to $\left(D^{2}, S^{1}\right)$, and hence is a P.D. pair. Moreover, since $\pi_{1}(B S G)=0$, we have that

$$
f \circ h: S^{1} \rightarrow X^{1} \rightarrow B S G
$$

is null homotopic. Hence $f \circ h$ extends to a map $\bar{f}: D^{2} \rightarrow B S G$. Let $g=\bar{f} \cup f: D^{2} \cup_{h} X^{1} \rightarrow B S G$, and $\bar{b}$ be the map of normal spherical fibre space covering $g$. Then $\left(X^{1}, f, b\right)$ becomes the boundary of $\left(\left(D^{2} \cup_{h} X^{1}, X^{1}\right), g, \bar{b}\right)$. This shows that $\left[\left(X^{1}, f, b\right)\right]=0$ in $\Omega_{1}^{\text {P.D. }}$. Hence $\Omega_{1}^{\text {P.D. }}=0$. So Theorem (A) is trivially true for $n=1$.

$$
n=2 \text {. Let }\left[\left(X^{2}, f, b\right)\right] \in \Omega_{2}^{\text {P.D. }} \text {. Let } h: X^{2} \rightarrow M^{2} \text { be an Eck- }
$$
mann et al. homotopy equivalence, where $M^{2}$ is some closed surface of genus $\geq 0$. This means that we have the following homotopy commutative diagram:

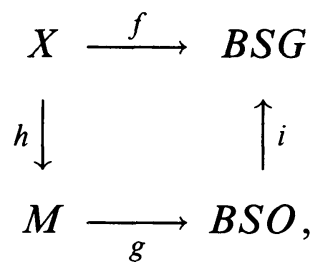

where $g$ classifies the stable normal bundle of $M$ and $i$ is the natural inclusion. Since $M$ is the boundary of an orientable 3-manifold $N^{3}$ (note that $\Omega_{2}^{\text {Diff }}=0$ ), we have the following commutative diagram:

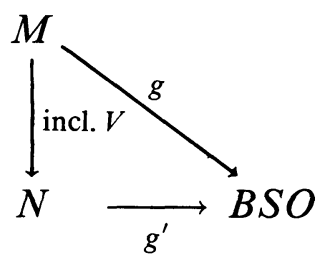

Now considering $M$ as the mapping cylinder of $h$ (up to homotopy) we have a homotopy inclusion $X \rightarrow N$, and if $\bar{b}$ represents the map of normal spherical fibrations covering $i \circ g^{\prime}$ then we have that $(X, f, b)$ 
is the boundary of $\left((N, X), i \circ g^{\prime}, \bar{b}\right)$. Hence $[(X, f, b)]=0$. Thus $\Omega_{2}^{\text {P.D. }}=0$. This proves Theorem (A) for $n=2$.

2. The Levitt exact sequence and a fundamental lemma. As we have mentioned in the introduction the transversality principle played an important role in the proof of existence of Rohlin's and Wall's exact sequences. This principle does not hold in P.D. and Levitt, Jones, Quinn [12], [11], [17] measures this failure with sufficient precision in the form of an exact sequence. With reference to a spherical fibration $\eta(E(\eta) \rightarrow B)$ this sequence can be written as:

$$
\begin{aligned}
\rightarrow L_{n}\left(\pi_{1}(B), w\right) \rightarrow T\left(S^{n+k}, T\left(\eta^{k}\right)\right) \rightarrow & \pi_{n+k}\left(T\left(\eta^{k}\right)\right) \\
& \rightarrow L_{n-1}\left(\pi_{1}(B), w\right) \rightarrow
\end{aligned}
$$

where $L_{i}\left(\pi_{1}(B), w\right)$ are the surgery obstruction groups of Wall [27], $w: \pi_{1}(B) \rightarrow \mathbb{Z} / 2$ being the homomorphism which sends an element $x$ in $\pi_{1}(B)$ nontrivially if $\eta \mid S^{1}$ is nontrivial for $S^{1} \rightarrow B$ representing $x$.

This sequence is natural with respect to the morphisms

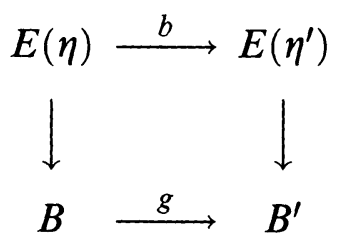

This means that the following diagram is commutative:

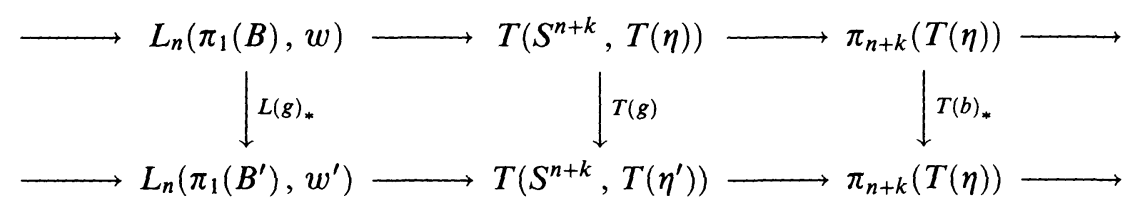
(see e.g. [2]).

We specialize to the case when

$$
\left\{\begin{array}{l}
\eta=\gamma_{B S G} \quad \text { and } \quad \eta^{\prime}=\gamma_{B G}, \quad B=B S G, \\
B^{\prime}=B G, \quad T(\eta)=M S G, \quad T\left(\eta^{\prime}\right)=M G . \\
g=\pi: B S G \rightarrow B G, \quad T(g)=r .
\end{array}\right.
$$

The proof of Theorem (A) for $n \geq 3$ will be based on an examination of (2.2) in the case when (2.3) holds and on the following fundamental lemma:

(2.4) Lemma. The sequence

$$
\pi_{*}(M S G) \stackrel{2}{\longrightarrow} \pi_{*}(M S G) \stackrel{T(b)_{*}}{\longrightarrow} \pi_{*}(M G)
$$

is exact at the middle term. 
Proof. Since $\pi_{*}(M G)$ is a $\mathbb{Z} / 2$-module (see [4]) the image of 2 is contained in the kernel of $T(b)_{*}$. Conversely, suppose $x \in \pi_{n}(M S G)$ satisfies $T(b)_{*}(x)=0$. We have to show that $x$ is divisible by 2 . It is known from $[4,16]$ that $M S G$ is homotopy equivalent to a wedge of Eilenberg-Mac Lane spectra, and the $\pi_{*}(M S G)$ are finite abelian groups whose 2 torsion parts are isomorphic to the homotopy groups of products of Eilenberg-Mac Lane spaces of type $\left(\mathbb{Z} / 2^{r_{\iota}}, n_{i}\right)$. That is, there is an isomorphism (with reference to the homotopy equivalence, say $f$, mentioned above):

$$
1 \otimes f_{*}: \pi_{n}(M S G)_{2} \stackrel{\simeq}{\longrightarrow} \pi_{n}\left(\underset{i}{\times} K\left(\mathbb{Z} / 2^{r}, n_{i}\right)\right),
$$

where $\pi_{n}(M S G)_{2}$ stands for $\pi_{n}(M S G)$ localized at 2. Now, if $x$ belongs to the odd torsion part of $\pi_{*}(M S G)$ then $T(b)_{*}(x)=0$ and also $x$ is divisible by 2 . So we need only to show that $1 \otimes f_{*}(x)$ is divisible by 2 (where $x$ is such that $T(b)_{*}(x)=0$ ). For this it is enough to show that for each $i, 1 \otimes f_{i *}(x)$ is divisible by 2 , where $1 \otimes f_{i *}$ is the homomorphism

$$
1 \otimes f_{i *}: \pi_{n}(M S G)_{2} \rightarrow \pi_{n}\left(K\left(\mathbb{Z} / 2^{r_{\iota}}, n_{i}\right)\right),
$$

(clearly $x \neq 0$ if and only if $n=n_{i}$ for some $i$ 's).

Let, for $k \gg n, f_{i}^{*}(l) \in H^{n_{i}+k}\left(M S G(k): \mathbb{Z} / 2^{r_{i}}\right)$ be the generator of the submodule isomorphic to $H^{n_{\imath}+k}\left(K\left(\mathbb{Z} / 2^{r_{\imath}}, n_{i}+k\right) ; \mathbb{Z} / 2^{r_{\imath}}\right)$, where $\imath$ is the fundamental cohomology class of $K\left(\mathbb{Z} / 2^{r}, r_{i}+k\right)$. Since $T(b)^{*}$ is onto in cohomology with $\mathbb{Z} / 2$ coefficients, there exists an element $y \in H^{n_{i}+k}(M G ; \mathbb{Z} / 2)$ such that $T(b)^{*}(y)=f_{i}^{*}(l) \bmod 2$.

Let $y=g_{i}^{*}(\bar{l})$ where

$$
g_{i}: M G(k) \rightarrow K\left(\mathbb{Z} / 2, n_{i}+k\right)
$$

is a map classifying $y$ and $\bar{l}$ is the fundamental class of $K\left(\mathbb{Z} / 2, n_{i}+k\right)$. If $\pi: K\left(\mathbb{Z} / 2^{r_{\imath}}, n_{i}+k\right) \rightarrow K\left(\mathbb{Z} / 2, n_{i}+k\right)$ is the map such that $\pi^{*}(\bar{l})=$ $l$ mod 2 then we have the following homotopy commutative diagram:

$$
\begin{aligned}
& M S G(k) \stackrel{f_{\imath}}{\longrightarrow} K\left(\mathbb{Z} / 2^{r}, n_{i}+k\right) \\
& \downarrow T(b) \quad \downarrow \pi \\
& M G(k) \stackrel{g_{\imath}}{\longrightarrow} K\left(\mathbb{Z} / 2, n_{i}+k\right) \text {. }
\end{aligned}
$$


This gives rise to a commutative diagram:

$$
\begin{aligned}
& \pi_{n_{i}+k}(M S G(k))_{2} \stackrel{1 \otimes f_{i *}}{\longrightarrow} \mathbb{Z} / 2^{r_{t}} \\
& \downarrow T(b) \text { * } \downarrow \pi_{*} \\
& \pi_{n_{l}+k}(M G(k))_{2} \stackrel{1 \otimes g_{l *}}{\longrightarrow} \mathbb{Z} / 2 .
\end{aligned}
$$

From the diagram (2.5) it follows that if $T(b)_{*}(x)=0$ then $1 \otimes f_{i_{*}}(x)$ is divisible by 2 . This finishes the proof of the lemma.

3. Proof of Theorem $(A)$ for $n \not \equiv 3(\bmod 4), n>3$. Diagram $(2.2)$ in the case of (2.3) becomes:

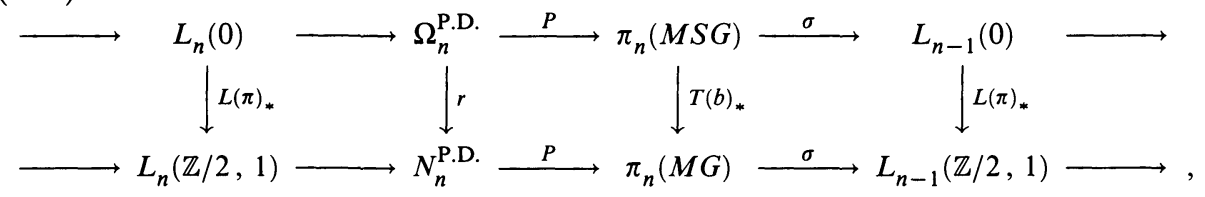

where

$$
\begin{gathered}
L_{n}(0)= \begin{cases}\mathbb{Z} & \text { if } n \equiv 0 \quad(\bmod 4), \\
\mathbb{Z} / 2 & \text { if } n \equiv 2 \quad(\bmod 4), \\
0 & \text { if } n \text { is odd }\end{cases} \\
L_{n}(\mathbb{Z} / 2,1)= \begin{cases}\mathbb{Z} / 2, & n \text { even } \\
0, & n \text { odd }\end{cases}
\end{gathered}
$$

We consider the following three cases:

I. $n \equiv(\bmod 4), n>3$. Then (3.1) and (3.2) give

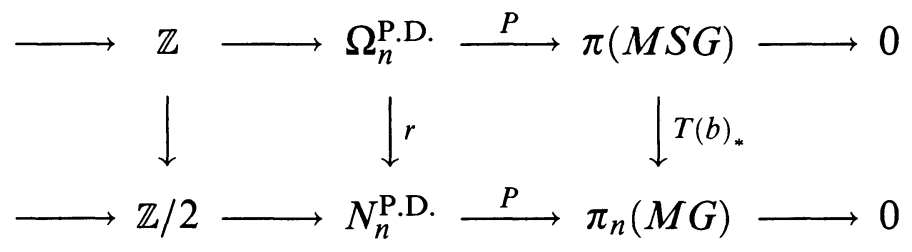

If $x \in \Omega_{n}^{\text {P.D. }}$ satisfies $r(x)=0$ then $P(x)$ is divisible by 2 . So there exists a $z \in \Omega_{n}^{\text {P.D. }}$ such that $2 P(z)=P(x)$, that is, $2 z-x \in \operatorname{Ker} P=$ $\operatorname{Im} L_{n}(0) \subseteq \Omega_{n}^{\text {P.D. }}$. By [3, Theorem 1.2] $\operatorname{Im} L_{n}(0)$ is generated by the Milnor manifold which is Poincaré cobordant to $8\left(\mathbb{C} P^{2}\right)^{k}$, if $n=4 k$ $\left(\mathbb{C} P^{2}\right.$ stands for complex projective space of dimension 2). Hence $x$ is divisible by 2 . This proves Theorem $(\mathrm{A})$ for $n \equiv 0(\bmod 4)$. 
II. $n \equiv 1(\bmod 4), n>3$. In this case $(3.1)$ and $(3.2)$ give

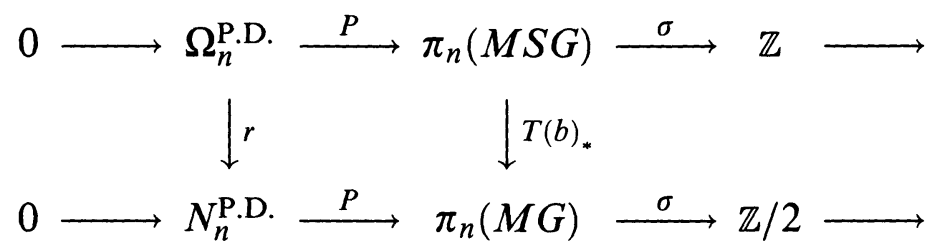

Since $\pi_{*}(M S G)$ is torsion $\pi_{n}(M S G) \stackrel{\sigma}{\longrightarrow} \mathbb{Z}$ is the zero map.

So $P: \Omega_{n}^{\text {P.D. }} \rightarrow \pi_{n}(M S G), n \equiv 1(\bmod 4)$, is an isomorphism. Theorem (A) now follows from Lemma (2.4).

III. $n \equiv 2(\bmod 4), n>3$. In this case $(3.1)$ and $(3.2)$ give:

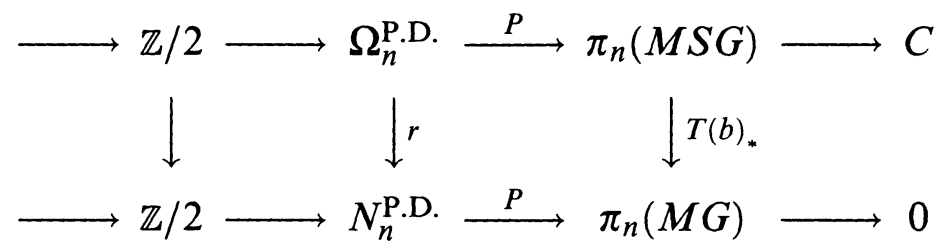

By a result of [2], $\pi_{4 k+3}(M S G) \stackrel{\sigma}{\longrightarrow} L_{4 k+2}(0)=\mathbb{Z} / 2$ is surjective. So by exactness $P$ is an isomorphism. Hence again Theorem (A) follows from Lemma $(2.4)$ for $n \equiv 2(\bmod 4)$.

Thus we have proved Theorem $(\mathrm{A})$ for $n \not \equiv 3(\bmod 4)$.

4. Proof of Theorem $(A)$ for $n \equiv 3(\bmod 4)$. We first prove the following proposition essentially due to Browder and Brumfiel [3]

(4.1) Proposition. The short exact sequence

$$
0 \rightarrow \Omega_{4 k+3}^{\text {P.D. }} \stackrel{P}{\longrightarrow} \pi_{4 k+3}(M S G) \stackrel{\sigma}{\longrightarrow} \mathbb{Z} / 2 \rightarrow 0
$$

is split exact for all integers $k \geq 0$.

Proof. We first show that $\pi_{3}(M S G)=\mathbb{Z} / 2$. For this consider the homotopy exact sequence of the pair $(M S G, M S O)$. Using the fact that $\pi_{i}(M S O)=0$ for $i=1,2,3$ and $\pi_{1}(M S G)=0$ we get that $\pi_{2}(M S G) \cong \pi_{2}(M S G, M S O)$. From the relative Hurewicz theorem we have $\pi_{2}(M S G, M S O) \cong H_{2}(M S G, M S O ; \mathbb{Z})$. Using the Thom isomorphism we get that $H_{2}(M S G, M S O ; \mathbb{Z}) \cong H_{2}(B S G, B S O ; \mathbb{Z})$.

Again from the relative Hurewicz theorem we have

$$
\mathrm{H}_{2}(B S G, B S O ; \mathbb{Z}) \cong \pi_{2}(B S G, B S O) \text {. }
$$


Now, comparing the homotopy exact sequence of the pair $(B S G, B S O)$ and that of the fibration

$$
G / O \rightarrow B S O \rightarrow B S G
$$

we get $\pi_{2}(B S G, B S O) \cong \pi_{1}(G / O)=0$ (see [22]). A similar procedure will give us that $\pi_{3}(M S G)=\mathbb{Z} / 2$.

We have thus proved (4.1) for $k=0$. We claim that the truth of (4.1) for $k=0$ implies truth of (4.1) for all $k>0$. For, note that the Whitney join of spherical fibrations gives rise to a map $\mu: M S G \wedge$ $M S G \rightarrow M S G$ and in particular to a map $\mu: M S G \wedge M S O \rightarrow M S G$.

Using the product formula for the transversality obstruction $\sigma$ : $\pi_{*}(M S G) \rightarrow \mathbb{Z} / 2$, as given in [7], we get the following commutative diagram:

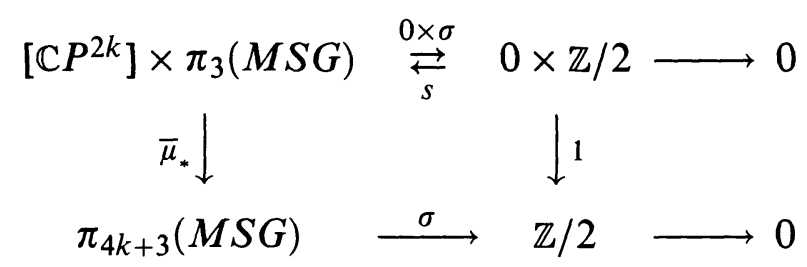

for all $k>0$, where we have identified the cobordism class of $\mathbb{C} P^{2 k}$ in $\Omega_{4 k}^{\text {Diff }}$ with its image in $\pi_{4 k}(M S O)$ under the Thom-Pontrjagin isomorphism, and $\bar{\mu}_{*}$ is the restriction of the composite:

$$
\pi_{4 k}(M S O) \times \pi_{3}(M S G) \rightarrow \pi_{4 k+3}(M S O \wedge M S G) \rightarrow \pi_{4 k+3}(M S G) .
$$

Now the top horizontal map of the above commutative diagram is split by the case for $k=0$, and hence the lower horizontal map $\sigma: \pi_{4 k+3}(M S G) \rightarrow \mathbb{Z} / 2$ is also split. This completes the proof of Proposition (4.1).

Proof of Theorem (A) for $n \equiv 3(\bmod 4)$. Using (3.1) and (3.2) we have:

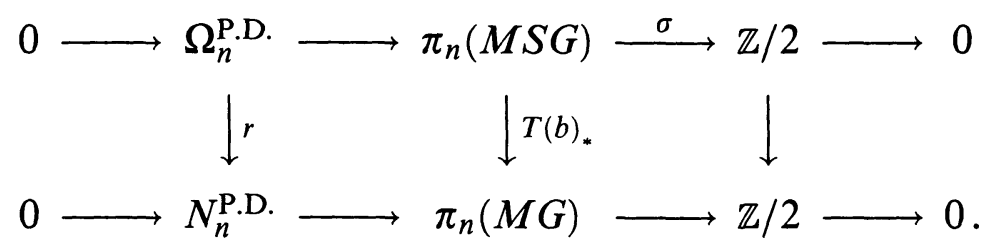

Let $x \in \Omega_{n}^{\text {P.D. }}$ satisfy $r(x)=0$. By Lemma (2.4) $P(x)$ is divisible by 2 , so there exists $y^{\prime} \in \pi_{n}(M S G)$ such that $P(x)=2 y^{\prime}$. If $y^{\prime} \in$ $\operatorname{Im} P$ then by the injectivity of $P, x=2 y$ for some $y \in \Omega_{n}^{\text {P.D. }}$. 
If $y^{\prime} \notin \operatorname{Im} P$ then $\sigma\left(y^{\prime}\right)=1$, and by (4.1) there is an element $z \in$ $\pi_{n}(M S G)-\operatorname{Im} P$ of order 2; hence $\sigma\left(z+y^{\prime}\right)=\sigma(z)+\sigma\left(y^{\prime}\right)=1+1=0$. So $z+y^{\prime} \in \operatorname{Im} P$ (by exactness), and hence there exists $y \in \Omega_{n}^{\text {P.D. }}$ such that $P(y)=z+y^{\prime}$. Now $P(2 y)=2\left(z+y^{\prime}\right)=2 z+2 y^{\prime}=2 y^{\prime}=P(x)$ and hence by injectivity of $P, x=2 y$. This proves Theorem (A) for $n \equiv 3(\bmod 4)$, and the proof of Theorem $(\mathrm{A})$ is complete.

\section{Existence of a homomorphism $\partial: \widetilde{N}_{n}^{\text {P.D. }} \rightarrow \Omega_{n-1}^{\text {P.D }}$.}

From the definition of the cobordism groups $\widetilde{N}_{n}^{\text {P.D. }}$ we have a Pontrjagin-Thom homomorphism

$$
P: \widetilde{N}_{n}^{\text {P.D. }} \rightarrow \pi_{n+k}\left(M S G(k-1) \wedge \mathbb{R} P^{2}\right), \quad k \gg n
$$

[21, pp. 148, 172], which fit into the following Levitt's exact sequence

$$
\begin{aligned}
\rightarrow L_{n}\left(1 \times \mathbb{Z}^{-}\right) \rightarrow \widetilde{N}_{n}^{\text {P.D. }} \stackrel{P}{\rightarrow} \pi_{n+k}(M S G(k-1) \wedge & \left.\mathbb{R} P^{2}\right) \\
& \stackrel{\hat{\sigma}}{\rightarrow} L_{n-1}\left(1 \times \mathbb{Z}^{-}\right) \rightarrow
\end{aligned}
$$

where

$$
L_{n}\left(1 \times \mathbb{Z}^{-}\right) \cong \begin{cases}\mathbb{Z} / 2 & \text { for } n \equiv 0(\bmod 4), \\ 0 & \text { for } n \equiv 1(\bmod 4), \\ \mathbb{Z} / 2 & \text { for } n \equiv 2(\bmod 4) \\ \mathbb{Z} / 2 & \text { for } n \equiv 3(\bmod 4) .\end{cases}
$$

See Wall [27] Brumfiel-Morgan [7].

(5.2) Proposition. P: $\widetilde{N}_{n}^{\text {P.D. }} \rightarrow \pi_{n+k}\left(M S G(k-1) \wedge \mathbb{R} P^{2}\right)$ is an injection $\forall n$.

Proof. For $n \equiv 1(\bmod 4) P_{n}$ is trivially injective.

For $n \equiv 0,2(\bmod 4)$ obstruction to transversality homomorphisms

$$
\hat{\sigma}: \pi_{n+1+k}\left(M S G(k-1) \wedge \mathbb{R} P^{2}\right) \rightarrow \mathbb{Z} / 2
$$

are given by cohomology classes $\widetilde{\mathscr{K}}$ and $\widetilde{\mathscr{L}}$ of Brumfiel-Morgan [7] and $\hat{\sigma}$ is nonzero by [10, Prop. 5.2].

For $n \equiv 3(\bmod 4)$ we note that

$$
\hat{\sigma}: \pi_{4 n+k}\left(M S G(k-1) \wedge \mathbb{R} P^{2}\right) \rightarrow \mathbb{Z} / 2
$$

is given by the composite

$$
\hat{\sigma}=\sigma \circ \partial: \pi_{4 n+k}\left(M S G(k-1) \wedge \mathbb{R} P^{2}\right)
$$

$$
\rightarrow \pi_{4 n-1+k-1}(M S G(k-1)) \stackrel{\sigma}{\rightarrow} \mathbb{Z} / 2
$$


(see Brumfiel-Morgan [7, p. 33]) and that $\sigma$ is nonzero (refer to $\S 4$ ). Also from the exact sequence

$$
\begin{aligned}
\rightarrow \pi_{4 n+k}\left(M S G(k-1) \wedge \mathbb{R} P^{2}\right) \stackrel{\partial}{\rightarrow} \pi_{4 n-1+k-1}(M S G(k-1)) \stackrel{\times 2}{\longrightarrow} & \rightarrow \pi_{4 n-1+k-1}(M S G(k-1)) \rightarrow
\end{aligned}
$$

one gets that $\partial$ is onto for $4 n-1=3\left(\right.$ as $\left.\pi_{3}(M S G)=\mathbb{Z} / 2\right)$ and hence $\hat{\sigma}: \pi_{4+k}\left(M S G(k-1) \wedge \mathbb{R} P^{2}\right) \rightarrow \mathbb{Z} / 2$ is onto.

To prove that, $\forall n>1, \hat{\sigma}: \pi_{4 n+k}\left(M S G(k-1) \wedge \mathbb{R} P^{2}\right) \rightarrow \mathbb{Z} / 2$ is onto one takes products of a preimage of 1 under $\hat{\sigma}$ in $\pi_{4+k}\left(M S G(k-1) \wedge \mathbb{R} P^{2}\right)$ with the homotopy class represented by $\left[\mathbb{C} P^{2(n-1)}\right]$, as was done in $\S 4$.

To define $\partial: \widetilde{N}_{n}^{\text {P.D. }} \rightarrow \Omega_{n-1}^{\text {P.D. }}$ look at the diagram:

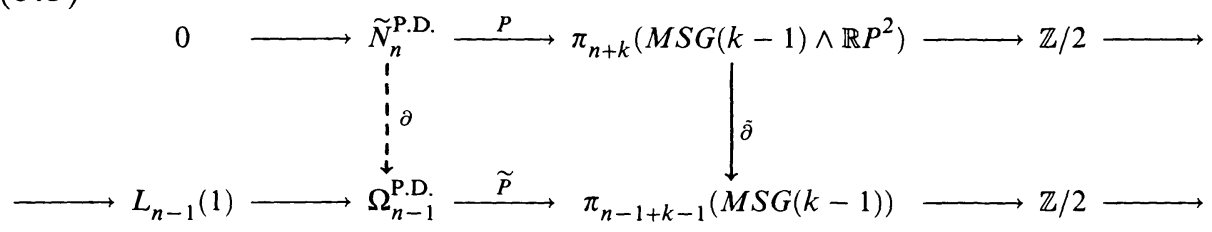

Let $x \in \tilde{N}_{n}^{\text {P.D. }}$. Look at $\tilde{\partial} \circ P(x)$. For $(n-1) \not \equiv 0,3(\bmod 4)$, $\widetilde{P}$ is an isomorphism, so $\exists$ a unique $y \in \Omega_{n-1}^{\text {P.D. }}$ s.t. $\widetilde{P}(y)=\tilde{\partial} \circ P(x)$. Define

$$
\partial(x)=y .
$$

For $(n-1) \equiv 3(\bmod 4)$, we have a splitting

$$
s: \pi_{n-1+k-1}(M S G(k-1)) \underset{\widetilde{P}}{\rightleftarrows} \Omega_{n-1}^{\text {P.D. }},
$$

see $\S 4$. So define

$$
\partial(x)=s(\tilde{\partial} \circ P(x)) .
$$

For $(n-1) \equiv 0(\bmod 4)$ lower horizontal sequence of $(5.3)$ has the form

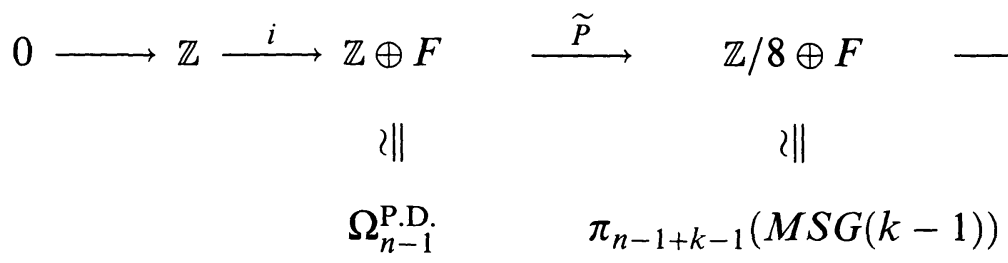

where $F$ is a torsion group (see Browder-Brumfiel [3]) where $i(1)=$ $\left(\left[8\left(\mathbb{C} P^{2}\right)^{l}\right], 0\right), 4 l=n-1 ; \mathbb{Z}$ summand of $\mathbb{Z} \oplus F$ is generated by $\left[\left(\mathbb{C} P^{2}\right)^{l}\right]$. 
Now $\tilde{\partial} \circ P(x) \in \mathbb{Z} / 8 \oplus F$. Take the projection $\pi_{2} \circ \tilde{\partial} \circ P(x)$ of $\tilde{\partial} \circ P(x)$ onto $F$. Since $\widetilde{P} / F: F \rightarrow F$ is identity we get a unique element $y \in \Omega_{n-1}^{P . D}$ such that $\widetilde{P}(y)=\pi_{2} \tilde{\partial} P(x)$. Define

$$
\partial(x)=y \text {. }
$$

(Justification of projecting $\tilde{\partial} \circ P(x)$ onto $F: \tilde{N}^{\text {P.D. }}$ being a torsion group no homomorphism from $\tilde{N}^{\text {P.D. }} \rightarrow \Omega^{\text {P.D. }}$ can take an element $x$ into the free part of $\Omega^{\text {P.D. }}$ nontrivially.)

This completes the definition of

$$
\partial: \tilde{N}_{n}^{\text {P.D. }} \rightarrow \Omega_{n-1}^{\text {P.D. }} \quad \forall n \geq 3 .
$$

For $n=1,2$ one can define a homomorphism using low dimensional classification of P.D. spaces (see $[26,9]$ ).

6. Proof of Theorem (B). This involves proving exactness of the following three sequences:

(i) $\Omega_{n}^{\text {P.D. }} \stackrel{2}{\longrightarrow} \Omega_{n}^{\text {P.D. }} \stackrel{r}{\longrightarrow} \tilde{N}_{n}^{\text {P.D. }}$,

(ii) $\tilde{N}^{\text {P.D. }} \stackrel{\partial}{\longrightarrow} \Omega_{n-1}^{\text {P.D. }} \stackrel{2}{\longrightarrow} \Omega_{n-1}^{\text {P.D }}$,

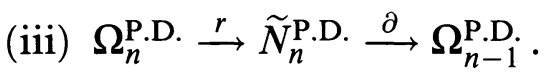

Exactness of (i). This follows from Theorem A, the fact that $\pi_{n+k}\left(M S G(k-1) \wedge \mathbb{R} P^{2}\right)$ is a $\mathbb{Z} / 2$-module, and that

$$
P: \tilde{N}_{n}^{\text {P.D. }} \rightarrow \pi_{n+k}\left(M S G(k-1) \wedge \mathbb{R} P^{2}\right) \text { is injective. }
$$

Exactness of (ii). $2 \circ \partial=0$ follows from the definition of $\partial$, the fact that $\partial(x)$ is a torsion element of $\Omega_{n-1}^{\text {P.D. }}$, and the fact that

$$
\begin{aligned}
\rightarrow \pi_{n+k}\left(M S G(k-1) \wedge \mathbb{R} P^{2}\right) \stackrel{\tilde{\partial}}{\rightarrow} & \pi_{n-1+k-1}(M S G(k-1)) \\
& \stackrel{\times 2}{\longrightarrow} \pi_{n-1+k-1}(M S G(k-1)) \rightarrow
\end{aligned}
$$

is exact.

Now let $x \in \Omega_{n-1}^{\text {P.D. }}$ such that $2(x)=0$.

Let $X^{n-1}$ be an oriented P.D. space representing $x$. That is $x=[(X, f, b)]$. Then there exists $((Y, \partial Y), g, c),(Y, \partial Y)$ an oriented P.D. pair with an orientation preserving homotopy equivalence of $\partial Y$ with $2 X$. Let $Z$ be a P.D. space obtained by identifying the two copies of $X$ in $Y$ so as to give a triple $(Z, \tilde{g}, \tilde{c})$, where $\tilde{g}, \tilde{c}$ are obtained from $g, c$ respectively by this identification. Let us define a continuous metric $\rho$ on $Y$ so that the distance of the 
two copies of $X$ in $Y$, say $X_{1}, X_{2}$ is $>1$ (since $Y$ is a finite CWcomplex it can be embedded in Euclidean space of sufficiently high dimension). Let us define a map $\lambda^{\prime}: Y \rightarrow[0,1]$ by

$$
\lambda^{\prime}(y)= \begin{cases}\rho\left(y, X_{1}\right) & \text { if } \rho\left(y, X_{1}\right)<1 / 2, \\ 1-\rho\left(y, X_{2}\right) & \text { if } \rho\left(y, X_{2}\right)<1 / 2, \\ 1 / 2 & \text { otherwise }\end{cases}
$$

as in [24].

Let $\lambda^{\prime}$ induce $\lambda: Z \rightarrow S^{1}$ on identifying $X_{1}, X_{2}$ and 0,1 . Then $\lambda^{-1}(0)=X \subseteq Z$. Since $Y \stackrel{g \times \lambda^{\prime}}{\longrightarrow} B S G \times[0,1]$ is homotopic to $g: Y \rightarrow B S G,\left(g \times \lambda^{\prime}\right)^{*}\left(\gamma \oplus \varepsilon^{0}\right)$ is fibre homotopy equivalent to $\nu_{Y}$. For $Z \stackrel{\tilde{g} \times \lambda}{\longrightarrow} B S G \times S^{1}$ therefore we have that $(\tilde{g} \times \lambda)^{*}\left(\gamma \oplus \gamma^{1}\right)$ is fibre homotopy equivalent to $\nu_{Z}$. So $w_{1}(Z)=w_{1}\left(\nu_{Z}\right)$ comes from an integral class. Therefore,

$$
[(Z, \tilde{g} \times \lambda, \tilde{c} \times \tilde{\lambda})] \in \tilde{N}_{n}^{\text {P.D }} .
$$

Now, since $\mathbb{Z} / 2$-spherical characteristic numbers of $X$ are determined by that of $Z$ (by a simple calculation similar to [Lemma 2, Wall [24]), and integral-spherical characteristic numbers as well as index of $X$ is zero ( $x$ being a torsion element), hence $(Z, \tilde{g} \times \lambda, \tilde{c} \times \tilde{\lambda})$ determines $(x, f, b)$ up to oriented cobordism by Theorem (D). So $x \in \operatorname{Im} \partial$.

Exactness of (iii). $\partial \circ r=0$, because we can take $\partial(r(x))$ represented by the empty manifold. Now let $x \in \widetilde{N}_{n}^{\text {P.D. such that }} \partial(x)=0$. Then by definition of $\partial, \tilde{\partial} \circ P(x)=\widetilde{P} \circ \partial(x)=0$ (refer to diagram (5.3)). So, from exactness of

$$
\begin{aligned}
\stackrel{\times 2}{\longrightarrow} \pi_{n+k-1}(M S G(k-1)) \stackrel{\tilde{r}}{\rightarrow} \pi_{n+k}\left(M S G(k-1) \wedge \mathbb{R} P^{2}\right) \\
\stackrel{\tilde{\partial}}{\rightarrow} \pi_{n+k-2}(M S G(k-1)) \rightarrow
\end{aligned}
$$

$\exists y \in \pi_{n+k-1}(M S G(k-1))$ such that $\tilde{r}(y)=P(x)$. Now $\widetilde{P}: \Omega_{n}^{\text {P.D }} \rightarrow$ $\pi_{n+l-1}(M S G(k-1))$ is onto for $n \not \equiv 3(\bmod 4)$. So for these $n \exists z \in$ $\Omega_{n}^{\text {P.D. such that }} \widetilde{P}(z)=y$, and hence $r(z)=x$ (using the commutative diagram

$$
\begin{aligned}
& \Omega_{n}^{\text {P.D. }} \stackrel{\widetilde{P}}{\longrightarrow} \pi_{n+k-1}(M S G(k-1)) \\
& r \downarrow \quad \downarrow \tilde{r} \\
& \left.N_{n}^{\text {P.D. }} \underset{\text { injective }}{\stackrel{P}{\longrightarrow}} \pi_{n+k}\left(M S G(k-1) \wedge \mathbb{R} P^{2}\right)\right) \text {. }
\end{aligned}
$$


For $n \equiv 3(\bmod 4)$ we appeal again to $[7$, p. 33] to get that $\sigma(y)=$ $\hat{\sigma} \circ \tilde{r}(y)=\hat{\sigma}(P(x))=0$ from (5.1).

So $y$ is in the image of $\widetilde{P}$; so $\exists z \in \Omega_{n}^{\text {P.D. with }} \widetilde{P}(z)=y$. So $r(z)=x$ as earlier.

7. Characterization of elements of Poincaré cobordism groups. In this section we shall prove Theorem (C) and Theorem (D). For this we shall first prove the following propositions.

(7.1) Proposition. A Poincaré transversal element

$$
\alpha \in \pi_{n+k}(M G(k))
$$

corresponding to an element $[X] \in N_{n}^{\mathrm{P} . D}$. is zero if and only if all $\mathbb{Z} / 2$-normal spherical characteristic numbers of $X$ are zero.

(7.2) Proposition. A Poincaré transversal element

$$
\alpha \in \pi_{n+k}(M S G(k))
$$

corresponding to an element $[X] \in \Omega_{n}^{\text {P.D. }}$ is zero if and only if all $\mathbb{Z} / 2$ as well as integral normal spherical characteristic numbers of $X$ are zero.

Proof of Propositions (7.1) and (7.2). Note first that since $M G$ and $M S G$ are of the same homotopy type as wedges of EilenbergMac Lane spectra [4], [16], the Hurewicz homomorphisms

$$
\begin{aligned}
& h_{*}: \pi_{n+k}(M G(k)) \rightarrow H_{n+k}(M G(k) ; \mathbb{Z} / 2) \text { and } \\
& h_{*}: \pi_{n+k}(M S G(k)) \rightarrow H_{n+k}(M S G(k) ; \mathbb{Z})
\end{aligned}
$$

are injective for all $n$ and $k$. This shows that every nonzero element $\alpha$ in $\pi_{n+k}\left(M S G(k)\right.$ ) (respectively in $\pi_{n+k}(M G(k))$ ) is detected by an element of $H^{n+k}(M S G(k) ; \mathbb{Z}$ ) (respectively of $\left.H^{n+k}(M G(k) ; \mathbb{Z} / 2)\right)$. This means that if $\alpha=[f], f: S^{n+k} \rightarrow$ $M S G(k)$ (or $\left.f: S^{n+k} \rightarrow M G(k)\right)$, then there exists an element $X \in$ $H^{n+k}(M S G(k) ; \mathbb{Z}) \quad$ (respectively $X \in H^{n+k}(M G(k) ; \mathbb{Z} / 2)$ ) such that $f^{*}(X) \neq 0$ in $H^{n+k}\left(S^{n+k} ; \mathbb{Z}\right)$ (respectively in $\left.H^{n+k}\left(S^{n+k} ; \mathbb{Z} / 2\right)\right)$.

Now, suppose $\alpha \in \pi_{n+k}(M S G(k))$ (respectively $\alpha \in \pi_{n+k}(M G(k))$ ) is Poincaré transversal in the sense of [2]. Then $\alpha=[f]$ is in the image of the Pontrjagin homomorphism $P: \Omega_{n}^{\text {P.D. }} \rightarrow \pi_{n+k}(M S G(k))$ (respectively $\left.P: N_{n}^{\text {P.D. }} \rightarrow \pi_{n+k}(M G(k))\right)$. 
Let $\alpha=P([(Y, g, b)])$.

Let $\nu_{Y}$ be the Spival normal fibre space over $Y, E\left(\nu_{Y}\right)$ the total space and $\bar{E}\left(\nu_{Y}\right)$ the mapping cylinder of the projection $E\left(\nu_{Y}\right) \rightarrow Y$. Then we have the following commutative diagram:

$$
\begin{array}{ccc}
H^{n+k}\left(\bar{E}\left(\nu_{Y}\right), E\left(\nu_{Y}\right) ; \mathbb{Z}\right) & \stackrel{\bar{b}^{*}}{ } H^{n+k}(\bar{E}(\gamma), E(\gamma) ; \mathbb{Z}) \\
\Phi^{*} \uparrow & \Phi^{*} \uparrow \\
H^{n}(Y, \mathbb{Z}) & \stackrel{g^{*}}{\longleftarrow} & H^{n}(B S G(k) ; \mathbb{Z})
\end{array}
$$

[respectively:

$$
\begin{array}{ccc}
H^{n+k}\left(\bar{E}\left(\nu_{Y}\right), E\left(\nu_{Y}\right) ; \mathbb{Z} / 2\right) & \stackrel{\bar{b}^{*}}{\longleftarrow} H^{n+k}(\bar{E}(\gamma) ; E(\gamma) ; \mathbb{Z} / 2) \\
\Phi^{*} \uparrow & \Phi^{*} \uparrow \\
H^{n}(Y, \mathbb{Z} / 2) & \stackrel{g^{*}}{\longleftarrow} & \left.H^{n}(B S G(k) ; \mathbb{Z} / 2)\right],
\end{array}
$$

where $\Phi^{*}$ stands for the Thom isomorphism, $\bar{E}(\gamma), E(\gamma)$ have the same meaning as $\bar{E}\left(\nu_{Y}\right), E\left(\nu_{Y}\right)$ respectively, and $\bar{b}$ is the map induced from $b$. We also have the fact that $f=\bar{b} \circ$ col. where col. is the natural collapsing map col. : $S^{n+k} \rightarrow T\left(\nu_{Y}\right)=\bar{E}\left(\nu_{Y}\right) / E\left(\nu_{Y}\right)$.

Now, since $\alpha=0$ if and only if $f^{*}=0$. Hence from the above commutative diagram it follows that $\alpha=0$ if and only if the composite $\Phi^{*} \circ g^{*}$ is zero, since the homomorphism $(\mathrm{col} .)^{n+k}$ is an isomorphism by definition of Spivak normal fibre space. Since $\Phi^{*}$ is an isomorphism we have that $\alpha=0$ if and only if $g^{*}$ is zero. This shows that $\alpha=0$ if and only if all normal spherical $\Lambda$-characteristic numbers of $Y$ are zero.

$$
\begin{aligned}
& \Lambda=\mathbb{Z} / 2 \text { and } \mathbb{Z} \quad \text { if }[(Y, g, b)] \in \Omega_{n}^{\text {P.D. }}, \\
& \Lambda=\mathbb{Z} / 2 \quad \text { if }[(Y, g, b)] \in N_{n}^{\text {P.D. }} .
\end{aligned}
$$

Completion of proofs of Theorem (C) and Theorem (D). We have seen that the Thom-Pontrjagin map $P: N_{n}^{\text {P.D. }} \rightarrow \pi_{n+k}(M G(k))$ is injective for all $n$, and the corresponding map $P \cdot \Omega_{n}^{\text {P.D. }} \rightarrow$ $\pi_{n+k}(M S G(k))$ is injective for all $n \not \equiv 0(\bmod 4)$ and for $n \equiv 0$ $(\bmod 4), \operatorname{Ker} P$ is generated by the class of $8\left(\mathbb{C} P^{2}\right)^{n / 4}$. Since the index of the intersection pairing of $\left(\mathbb{C} P^{2}\right)^{n / 4}$ denoted $I\left(\left(\mathbb{C} P^{2}\right)^{n / 4}\right)$ is equal to 1 , hence $I\left(8\left(\mathbb{C} P^{2}\right)^{n / 4}\right)=8$. 
Using these observations and Propositions (7.1), (7.2) the Theorems (C) and (D) follow.

\section{REFERENCES}

[1] W. Browder, Poincaré spaces their normal fibrations and surgery, Invent. Math., 17 (1972), 191-202.

[2] _ - The Kervaire Invariant, Products and Poincaré Transversality, Topology, 12 (1973), 145-158.

[3] W. Browder and G. Brumfiel, A note of cobordism of Poincaré duality spaces, Bull. Amer. Math. Soc., 77 (3) (1971), 400-403.

[4] W. Browder, A. Lieulevicius, and F. P. Peterson, Cobordism theories, Ann. Math., 84 (1966), 91-101.

[5] E. H. Brown, Jr. and F. P. Peterson, Relations among Characteristic classes I, Topology, 3 Supplement 1 (1964), 39-52.

[6] G. Brumfiel and J. Milgram, Normal maps, covering spaces, and quadratic functions, Duke Math. J., 44 (3) (1977), 663-694.

[7] G. Brumfiel, J. Milgram and J. Morgan, Homotopy theoretic consequence of Levitt's obstruction theory to transversality for spherical fibrations, Pacific J. Math., 67 (I) (1976), 1-100.

[8] P. E. Conner and E. E. Floyd, Differentiable Periodic Maps, Springer-Verlag, Ergn. Math., Band 33, 1964.

[9] Beno Eckmann and Peter Linnell, Poincaré duality groups of dimension two, II, Comment. Math. Helv., 58 (1983), 111-114.

[10] I. Hambleton and J. Milgram, Poincaré transversality for double covers, Canad. J. Math., 30 (6) (1978), 1319-1330.

[11] L. Jones, Patch spaces: A geometric representation for Poincaré spaces, Ann. Math., 97 (2) (1973), 306-343.

[12] N. Levitt, Poincaré duality cobordism, Ann. Math., 96 (2) (1972), 211-244.

[13] R. J. Milgram, The mod 2 spherical characteristic class, Ann. Math., 92 (2) (1970), 238-261.

[14] H. K. Mukerjee, Cobordism de Poincaré et un théorème de Rohlin, C. R. Acad. Sc. Paris, t Serie I 297 (1983), 345-347.

[15] __ La suite exacte de Wall dans le Cobordism de Poincaré, C. R. Acad. Sc. Paris, Serie I, t. 299, 33-36.

[16] F. P. Peterson and H. Toda, On the structure of $H^{*}(B S F ; \mathbb{Z} / p)$, J. Math. Kyoto Univ., 7-2 (1967), 113-121.

[17] F. Quinn, Surgery on Poincaré and normal spaces, Bull. Amer. Math. Soc., 78 (1972), 262-267.

[18] V. A. Rohlin, Intrinsic homologies, Dokl. Akad. Nauk S.S.S.R., 89 (1953), 789792.

[19] M. Spivak, Spaces satisfying Poincaré duality, Topology, 6 (1967), 77-102.

[20] J. Stasheff, A classification theorem for fibre spaces, Topology, 2 (1963), 239246.

[21] R. E. Stong, Notes on Cobordism Theory, Princeton University Press.

[22] D. Sullivan, Geometric Topology Seminar Notes, M.I.T., 1967

[23] R. Thom, Quelques propertetes globales des varietes differentiables, Commt. Math. Helv., 28 (1954), 17-86.

[24] C. T. C. Wall, Determination of the cobordism ring, Ann. Math., 72 (1960), 292-311. 
[25] C. T. C. Wall, Cobordism exact sequences for differentiable and combinatorial manifolds, Ann. Math., 77 (1963), 1-15.

[26] _-, Poincaré Complexes I, Ann. Math., 86 (1967), 213-245.

[27] _ Surgery on Compact Manifolds, Academic Press, London, New York (1970).

Received August 27, 1986 and in revised form June 1, 1989.

NORTH-EASTERN HILl UNIVERSITY

BIJNI COMPLEX, LAITUMKHRAH

Shillong-793003, Meghalaya

INDIA 



\section{PACIFIC JOURNAL OF MATHEMATICS EDITORS}

\author{
V. S. VARADARAJAN \\ (Managing Editor) \\ University of California \\ Los Angeles, CA 90024-1555-05 \\ Herbert Clemens \\ University of Utah \\ Salt Lake City, UT 84112 \\ Thomas ENRIGHT \\ University of California, San Diego \\ La Jolla, CA 92093
}

\section{R. FINN}

Stanford University

Stanford, CA 94305

Hermann FlaschKa

University of Arizona

Tucson, AZ 85721

VAUGHAN F. R. Jones

University of California

Berkeley, CA 94720

STEVEN KeRCKHOFF

Stanford University

Stanford, CA 94305
C. C. MOORE

University of California

Berkeley, CA 94720

Martin ScharlemanN

University of California

Santa Barbara, CA 93106

HAROLd STARK

University of California, San Diego

La Jolla, CA 92093

\section{ASSOCIATE EDITORS}
R. ARENS
E. F. BECKENBACH
B. H. NeumanN
F. Wolf
K. YoshidA (1906-1982)
(1904-1989)

\section{SUPPORTING INSTITUTIONS}
UNIVERSITY OF ARIZONA
UNIVERSITY OF BRITISH COLUMBIA
CALIFORNIA INSTITUTE OF TECHNOLOGY
UNIVERSITY OF CALIFORNIA
MONTANA STATE UNIVERSITY
UNIVERSITY OF NEVADA, RENO
NEW MEXICO STATE UNIVERSITY
OREGON STATE UNIVERSITY
UNIVERSITY OF OREGON
UNIVERSITY OF SOUTHERN CALIFORNIA
STANFORD UNIVERSITY
UNIVERSITY OF HAWAII
UNIVERSITY OF TOKYO
UNIVERSITY OF UTAH
WASHINGTON STATE UNIVERSITY
UNIVERSITY OF WASHINGTON 


\section{Pacific Journal of Mathematics}

Vol. 146, No. $1 \quad$ November, 1990

Primo Brandi and Anna Salvadori, A quasi-additivity type condition and

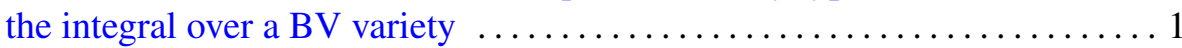

Dong M. Chung, Chull Park and David Lee Skoug, Operator-valued

Feynman integrals via conditional Feynman integrals ..............21

Paul Jolissaint, Index for pairs of finite von Neumann algebras . .........43

Miodrag Mateljević and Miroslav Pavlović, Multipliers of $H^{p}$ and

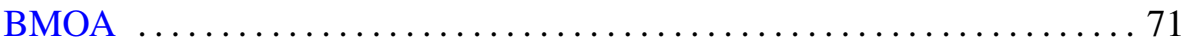

Himadri Kumar Mukerjee, Poincaré cobordism exact sequences and

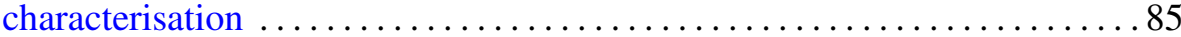

Thomas H. Otway, The coupled Yang-Mills-Dirac equations for differential

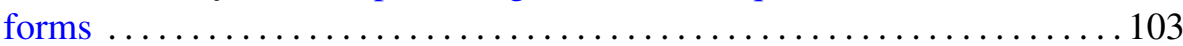

Sechiko Takahashi, Nevanlinna parametrizations for the extended

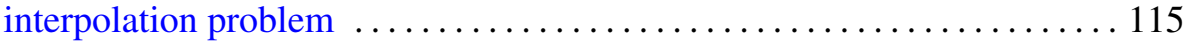

P. C. Trombi, Uniform asymptotics for real reductive Lie groups ........ 131 